\title{
Collectivity-induced quenching of signatures for shell closures
}

\author{
M. Bender, ${ }^{1,2}$ G. F. Bertsch, ${ }^{3}$ and P.-H. Heenen ${ }^{4,5}$ \\ ${ }^{1}$ Université Bordeaux, Centre d'Etudes Nucléaires de Bordeaux Gradignan, UMR5797, F-33175 Gradignan, France \\ ${ }^{2}$ CNRS/IN2P3, Centre d'Etudes Nucléaires de Bordeaux Gradignan, UMR5797, F-33175 Gradignan, France \\ ${ }^{3}$ Department of Physics and Institute for Nuclear Theory, \\ Box 351560, University of Washington, Seattle, WA 98195 \\ ${ }^{4}$ Service de Physique Nucléaire Théorique, Université Libre de Bruxelles, C.P. 229, B-1050 Bruxelles, Belgium \\ ${ }^{5}$ Physics Division, Argonne National Laboratory, \\ 9700 S. Cass Avenue, Argonne, IL 60439, U.S.A.
}

(Dated: 27 August 2008)

\begin{abstract}
Mass differences are an often used as signature and measure for shell closure. Using the angularmomentum projected Generator Coordinate Method and the Skyrme interaction SLy4, we analyze the modification of mass differences due to static deformation and dynamic fluctuations around the mean-field ground state.

PACS numbers: 21.10.Dr, 21.60.Jz,
\end{abstract}

\section{INTRODUCTION}

How the magic numbers that are well established along the line of stability evolve when moving away towards nuclei with large neutron or proton excess is still an unsettled question. Major recent experimental progress now allows one to follow magic numbers over long isotopic or isotonic chains far from stability. The data for many observables point to the disappearance of well established magic numbers and the appearance of new ones in nuclei which sometimes are still close to stability, and far from the drip lines.

From a theoretical point of view, the situation is still unclear. The interactions used in shell model calculations have had to be revised in order to be able to reproduce the new experimental data and to describe the evolution of shells, especially in nuclei in the $p f$ shell $1,2,2,3,4,4,5$, 6].

An analysis in terms of the self-consistent mean-field method performed well before most experimental data became available [7] has shown that, when approaching the drip lines, the increasing diffuseness of the nuclear surface for neutrons and the interaction between bound orbitals and the continuum affect the shape of the mean-field potentials of protons and neutrons. As a consequence, shell structure is modified, known gaps are quenched, and new ones might open. However, the coupling between bound orbitals and the continuum can be expected to be an important mechanism for nuclei in the immediate vicinity of the drip line only, when the Fermi energy is approaching zero. With the exception of the case of the lightest nuclei with mass below 30, there are no experimental data for such nuclei, and it is not obvious that the coupling with the continuum is an effect which has an impact on the known anomalies in shell structure. By contrast, the diffuseness of the neutron density might play a role in unstable nuclei, mainly through a reduction of the spin-orbit potentials for both protons and neutrons [8, 9]. However, this effect can be expected to appear gradually, and cannot not be respon- sible for those anomalies in shell structure where nuclear structure is suddenly changing when adding or removing a few nucleons only.

There are several observables that are used as possible signatures to put the evolution of shell closures in evidence when following isotopic or isotonic chains. From a theoretical point of view, the simplest one is the twonucleon separation energy in even-even nuclei, which can be easily calculated from the total binding energy. A jump in the two-nucleon separation energy is a direct indication of a sudden increase in the ground-state binding energy of a given nucleus, and often has a shell closure as its origin. Other quantities that are frequently analyzed and which are not directly related to the groundstate properties, but to the gaps that separate the different shells are provided by excitation spectra in even and odd-mass nuclei. At least in the context of mean-fieldbased models [10], these are more difficult to calculate and have larger associated theoretical uncertainties. Still, any conclusion about the evolution of shell structure requires clear clear trends from a variety of observables that probe complementary aspects. In particular, it should be supported also by data on electromagnetic moments and transition probabilities which provide information on the structure of the ground state and the excited-state wave functions.

Although there is no formal justification for this practice, the discussion of shell evolution is often conducted by theorists and experimentalists alike as if the following three quantities were equivalent:

1. single-particle energies in a spherical self-consistent mean field. They are then the eigenvalues of a single-particle Hamiltonian and the corresponding wave function is a single Slater determinant, i.e. a pure state;

2. effective single-particle energies as defined within the interacting shell model. They should in principle result from an average over a huge number of configurations $11,12,13,14,15,16,17]$; 
3. two-nucleon separation energies.

The last quantity is the only one which can be directly related to experimental data. Let us recall its definition:

$$
\begin{aligned}
& S_{2 p}(Z, N)=E(Z-2, N)-E(Z, N) \\
& S_{2 n}(Z, N)=E(Z, N-2)-E(Z, N),
\end{aligned}
$$

where $E(Z, N)$ is the total binding energy of the nucleus $Z, N$.

In the highly idealized pure HF case where the Koopman theorem is valid [18, 19], the two-nucleon separation energy is equal to -2 times the energy of the doublydegenerate orbital occupied by the two additional nucleons. This is never exactly the case in nuclear physics. First, pairing effects require a change in the energy of the Fermi level, resulting through self consistency to a change of all orbits. Even without pairing correlations, the two extra particles have an effect on the core and may induce at least a small rearrangement of all the orbits, or in the worst case, the appearance of deformations.

It is the purpose of this paper to analyze the role of static and dynamical quadrupole correlations on the systematics of $S_{2 q}(Z, N)$ in the context of mean-field based methods. Our analysis relies on the results of Ref. [20, 21] where systematic calculations of the masses of even-even nuclei were presented. The method allows one to introduce the correlations due to symmetry restorations (particle number and angular momentum) performed on axial mean-field configurations and to add a mixing of the projected configurations with respect to the axial quadrupole moment in the framework of the discretized Generator Coordinate Method (GCM). Since the starting point of the method is a mean-field calculation, the results give access at the same time to the spherical single-particle spectra of all even-even nuclei and to the two-nucleon separation energy of states including beyond mean-field correlations.

In the next section, we recall the main features of our method. We then discuss in details the evolution of the two-nucleon separation energy along $Z$ or $N=50$. Finally, we compare the results obtained for all isotopic and isotonic chains with the three different steps of our method to the experimental data.

\section{THE MODEL}

The method used to calculate binding energies for the ground states of even nuclei is described in detail in Refs. [20, 21] (referred to as paper I in the following). In this analysis, we use the energies as tabulated in [22].

To summarize the key features of our method, its starting point is a set of mean-field calculations including a constraint on the axial quadrupole moment. As effective interaction we employ a Skyrme energy density functional, the SLy4 parametrization 23], for the mean-field channel, and a density-dependent, zero-range interaction in the pairing channel. Two sets of correlations beyond the mean-field are introduced. First, the deformed wave functions are projected on both fixed particle numbers and on angular momentum. This projection corresponds to mixing degenerate mean-field wave functions which differ by a spatial rotation and to introduce fluctuations around the orientation of the mean-field. In a collective model terminology, these correlations would be called rotational correlations. A second step of our method consists in the mixing of projected mean-field wave functions as a function of their axial quadrupole moment. This corresponds to a vibrational correction in the collective model language. Our final wave function has the form:

$$
|J M \nu\rangle=\sum_{q} f_{J, \nu} \hat{P}_{M 0}^{J} \hat{P}_{N} \hat{P}_{Z}|q\rangle .
$$

The ket $|q\rangle$ is a (paired) mean-field state of axial quadrupole deformation $q$. The particle numbers and angular momentum quantum number are restored thanks to the operators $\hat{P}_{N}$ and $\hat{P}_{Z}$ which project on good neutron and proton numbers, and $\hat{P}_{M 0}^{J}$ which projects on angularmomentum $J$ with the $z$ component $M$ in the laboratory frame [24]. The projected wave functions are labeled by the index $q$ which indicates from which mean-field state they are obtained, although an intrinsic quadrupole moment has no direct physical meaning for a wave function with good angular momentum and can only be defined with the help of the collective model, in the same way as it is from experimental data. The weights $f_{J, \nu}(q)$ defining the mixing of the projected wave functions with respect to $q$ are obtained by variation of the total energy.

The mean-field wave functions are generated by treating the pairing correlations using the Lipkin-Nogami (LN) prescription. This enforces the presence of pairing in all nuclei and for all quadrupole moments, even at magic numbers, which is necessary to ensure the continuity of the mean-field wave functions required to perform the configuration mixing. Binding energies, however, are always recalculated after a projection on particle number [21].

We stress that there are no assumptions made in the model about the amplitude of the quadrupole fluctuations introduced into the calculations. Depending on the structure of a nucleus, this amplitude either corresponds to a small vibration around a pronounced minimum, to a large-amplitude motion in a soft and wide potential well, or to the mixing of several states around coexisting minima in the deformation energy surface. Let us recall also that our method is fully variational and that the energy of the ground state is lowered at each successive stage of the calculation.

Finally, one must note that the Skyrme interaction is not a force, but an energy density functional. It requires some care to be used beyond a mean-field approach, and there are still some open questions concerning this longstanding practice. The density-dependent terms are generalized using the standard prescription that the density is replaced by the transition density in the densitydependent terms. This is the only prescription that 
guarantees various consistency requirements of the energy functional [25, 26]. However, this procedure may lead to problems that have been put into evidence recently [27, 28, 29]. The numerical procedure that we have used (see Paper I for details) appears to be safe in this respect and no dramatic signs of problems have shown up when varying the number of discretization points in deformation. In any case, since we are interested here in trends as a function of $N$ and $Z$, errors of the order of 200 to $300 \mathrm{keV}$ would not affect our conclusions.

We will now compare results obtained from three wave functions that successively add quadrupole correlations:

1. spherical mean field states $|q=0\rangle$;

2. the mean-field minimum in the space of axial reflection-symmetric deformations $\left|q_{\text {min }}\right\rangle$, which might be spherical;

3. the ground state obtained after configuration mixing by the generator coordinate method of $J=0$ projected axial quadrupole. We refer to these wave functions in the following as projected GCM.

\section{RESULTS}

We will first illustrate the key points of the analysis of two-nucleon separation energies as measures of shell closures for the proton $Z=50$ and neutron $N=50$ shells. In a second step, we will then analyze the global systematics of two-nucleon separation energies for eveneven nuclei across the nuclear chart.

Recent experimental progress has provided many new direct, high-precision measurements of nuclear masses [30, 31], which allow comparison of calculations with data along chains that extend to exotic nuclei. Experimental masses used here are either taken from AME2003 32], where we exclude extrapolated values, or from Ref. 30, 31] when error bars are smaller or when the AME2003 values from indirect measurements do not agree with new directly measured ones.

\section{A. The $Z=50$ isotopic chain}

Figure 1 illustrates the difficulties encountered when relating two-nucleon separation energies to the gaps in the single-particle spectrum with the example of the chain of even-even Sn, $Z=50$, and Te, $Z=52$, isotopes. The lower panel shows the evolution of the eigenvalues of the spherical mean-field single-particle Hamiltonian $\epsilon_{p}$. Note that all $\mathrm{Sn}$ isotopes have a spherical ground state at the mean-field level.

The open blue circles, plotted in both panels, represent the two-proton separation energies $S_{2 p}$ for $N=50$ and $N=52$ from spherical mean-field calculations.

The magnitude of the $Z=50$ gap in the single-particle spectrum is fairly independent of $N$ in this case. The

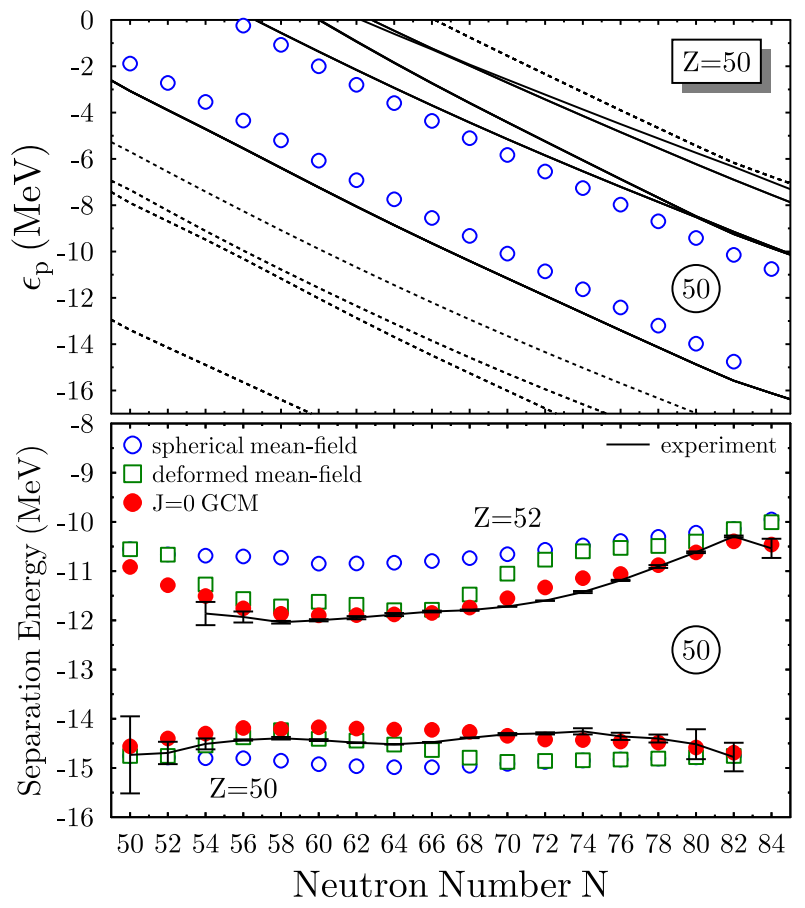

FIG. 1: (Color online) Top: Single-particle spectra of the protons for the chain of $\operatorname{Sn}(Z=50)$ isotopes and $-1 / 2$ times the two-proton separation energy for the $Z=50$ and $Z=52$ isotopic chains (see text). Bottom: Comparison of the gaps between $-S_{2 p}(Z=50, N) / 2$ and $-S_{2 p}(Z=52, N) / 2$ obtained at three levels of approximation with the experimental data. A linear trend equal to $(N-82)\left[S_{2 p}(Z=50, N=\right.$ $\left.50)-S_{2 p}(Z=50, N=82)\right] / 2$ using the spherical mean-field $S_{2 p}$ values has been subtracted from all $-S_{2 p}(Z, N) / 2$, such that the distance between the curves for a given $N$ is unchanged. In particular, the plotted values at $N=82$ are not affected, and the plotted spherical mean-field values for $(Z=50, N=50)$ and $(Z=50, N=82)$ are identical.

spacing between the $-S_{2 p} / 2$ for $Z=50$ and 52 is a poor measure of the true shell gap, even when $-S_{2 p} / 2$ values and the single-particle levels are calculated in the same framework and the same shape is enforced for all nuclei. Indeed, the $-S_{2 p} / 2$ spacing turns out to be smaller for all $N$ values. This result can be partly attributed to our treatment of pairing by the LN prescription. This method has the effect of moving the single-particle states away from the Fermi level. However, the amplitude of this change is of the order of 200 to $300 \mathrm{keV}$ at most and does not account for the difference between the single particle levels and $-S_{2 p} / 2$.

Still, the evolution with $N$ of the spherical mean-field $-S_{2 p} / 2$ values follows closely that of the single-particle levels: the difference between their values for $Z=50$ and $Z=52$ is nearly constant.

In the lower panel of this Fig. 1, the $-S_{2 p} / 2$ values for both isotopic chains are shown for spherical, deformed, and projected GCM calculations together with the experimental data. A global descending linear trend with $N$ has been taken out. The $-S_{2 p} / 2$ values from spherical 


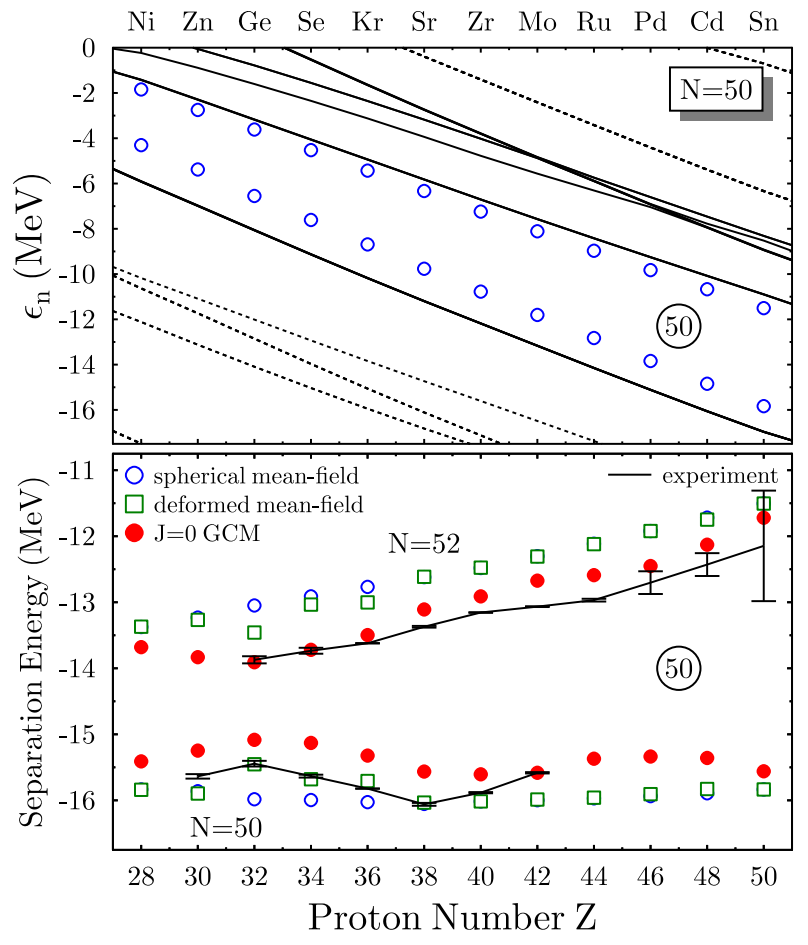

FIG. 2: (Color online) Same as Fig. 1 but for neutrons. In the lower panel, a global descending trend $(N-50)\left[S_{2 n}(Z=\right.$ $\left.28, N=50)-S_{2 n}(Z=50, N=50)\right] / 2$ using the spherical mean-field $S_{2 n}$ values has been subtracted from all $-S_{2 n}(Z, N) / 2$. The overall reduction of the distance between $S_{2 n}(Z, N=50)$ and $S_{2 n}(Z, N=52)$ when approaching $Z=28$ is clearly visible.

mean-field calculations differ the most from the actual data. They are smaller for $Z=50$ and larger for $Z=52$ than the data. In particular, the distance between the experimental $S_{2 p}$ values at $Z=50$ and at $Z=52$ is not constant; it decreases when going away from $N=82$, and starts to grow when approaching $N=50$.

A significant improvement is achieved by allowing the mean-field to deform. As outlined in paper I, some of the $\mathrm{Cd}(Z=48)$ and $\mathrm{Te}(Z=52)$ isotopes gain up to $2 \mathrm{MeV}$ in binding energy thanks to deformation, and this increases $S_{2 p}$ for $Z=50$ and decreases it for $Z=52$. Despite this effect of deformation, none of the nuclei involved in the calculation of the $S_{2 p}$ can be classified as well-deformed: they exhibit a transitional pattern, with soft deformation energy surfaces, and present shallow deformed minima in some cases. This soft topography has a significant effect when the fluctuations around the meanfield minima are taken into account. The $\mathrm{Cd}$ and $\mathrm{Te}$ isotopes are usually softer than the Sn ones for a given $N$-value, in such a way that $\mathrm{Cd}$ and Te gain more dynamical correlation energy. This increases the $S_{2 p}$ values for $Z=50$, but reduces them for $Z=52$.

Specifically, for these two isotopic chains, the change in the $S_{2 p}$ energies brought about by the dynamical quadrupole correlation energy is often large when the change due to the static deformation energy is small and vice versa. The resulting effect is that the projected GCM curves are much smoother than the deformed mean-field ones, and closely follow the experimental data. The only discontinuity left in Fig. 1 is at the neutron shell closure $N=82$, where the distance between $S_{2 p}(N, Z=50)$ and $S_{2 p}(N, Z=52)$ is largest, a phenomenon coined as "mutually enhanced magicity" in the literature 33, 34, 35]. From this result one can conclude that, within the present theoretical framework, the variation of the gap in the $S_{2 p}$ values as a function of $N$ is not related to a change in the underlying spherical shell structure, but to the variation of the energy contribution brought by static and dynamic quadrupole correlations to the masses of the nuclei that enter the calculation of $S_{2 p}$. The same qualitative behavior has been found for proton shells at $Z=82$ in Refs. [20, 21, 36].

\section{B. The $N=50$ isotopic chain}

The same analysis for the chains of $N=50$ and 52 isotones is performed in Figure 2. Recent mass measurements [31] allow one to follow $S_{2 n}$ down to $Z=30$. Qualitatively, the results are quite similar to those discussed for the $Z=50$ and 52 chains above, with two notable exceptions. First, the size of the $N=50$ gap in the singleparticle spectrum is slowly and continuously decreasing from $6.1 \mathrm{MeV}$ in ${ }^{100} \mathrm{Sn}$ to $4.4 \mathrm{MeV}$ in ${ }^{78} \mathrm{Ni}$, which constitutes "real" shell quenching and is a consequence of the increasing effect of the surface on neutron levels that are pushed up in the potential well. The spin-orbit splitting between the neutron $g_{9 / 2^{+}}$and $g_{7 / 2^{+}}$levels is slightly increased in our calculation when going from ${ }^{100} \mathrm{Sn}$ down to ${ }^{84} \mathrm{Ge}$, the lightest isotone where both are bound. The striking difference with respect to the protons in the Sn chain discussed above can be related to the impact of the Coulomb barrier: its presence for protons suppresses the diffuseness of the wave functions. Second, several fluctuations are superimposed on this smooth global trend, one of which slightly opens the gap at the proton sub-shell closures $Z=38$ and $Z=40$ and closes it for smaller $Z$ values, such that the calculated gap is smallest for $Z=32$, in agreement with experiment [31], when taking also the experimental $S_{2 n}$ value of the odd nucleus $(Z=31, N=52)$ into account.

A comparison of predictions of various mean-field and other mass models for the $N=50$ isotonic chain with the most recent data can be found in Ref. [31].

\section{Global systematics}

Figure 3 provides the two-proton separation energy $S_{2 p}$ for isotonic chains (now without the factor $-1 / 2$ ) in all but the lightest even-even nuclei calculated in 20, 21]. Together with the experimental data, the plots give the 


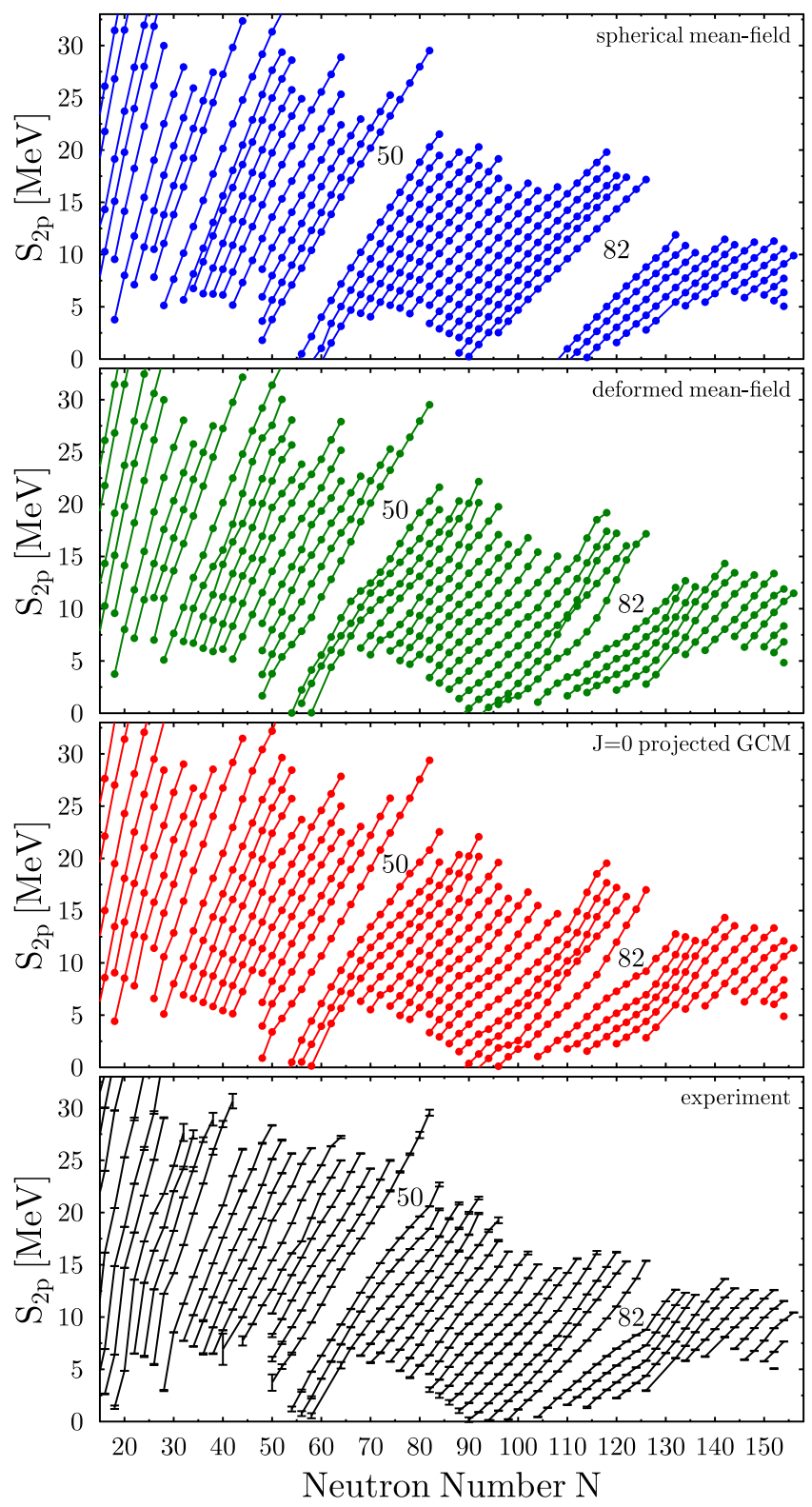

FIG. 3: (Color online) Two proton-separation energy $S_{2 p}$ for even-even nuclei. Lines connect nuclei in isotonic chains.

results for spherical and deformed mean-field calculations and for the projected GCM ground states.

The $S_{2 p}$ energies from spherical mean-field calculations (top panel) nearly follow straight lines, with a slope that slowly decreases with mass. Three gaps, corresponding to the magic numbers $Z=28,50$ and 82 , are clearly visible. Their widths do not vary significantly with neutron number. Most importantly, there is no sign of shell quenching in the $S_{2 p}$ trends, consistent with the gaps in the single-particle spectra that remain fairly constant as well.

The inclusion of static mean-field deformations has a significant effect on the curves. Compared to the spherical results, the magic gaps in the $S_{2 p}$ values are reduced

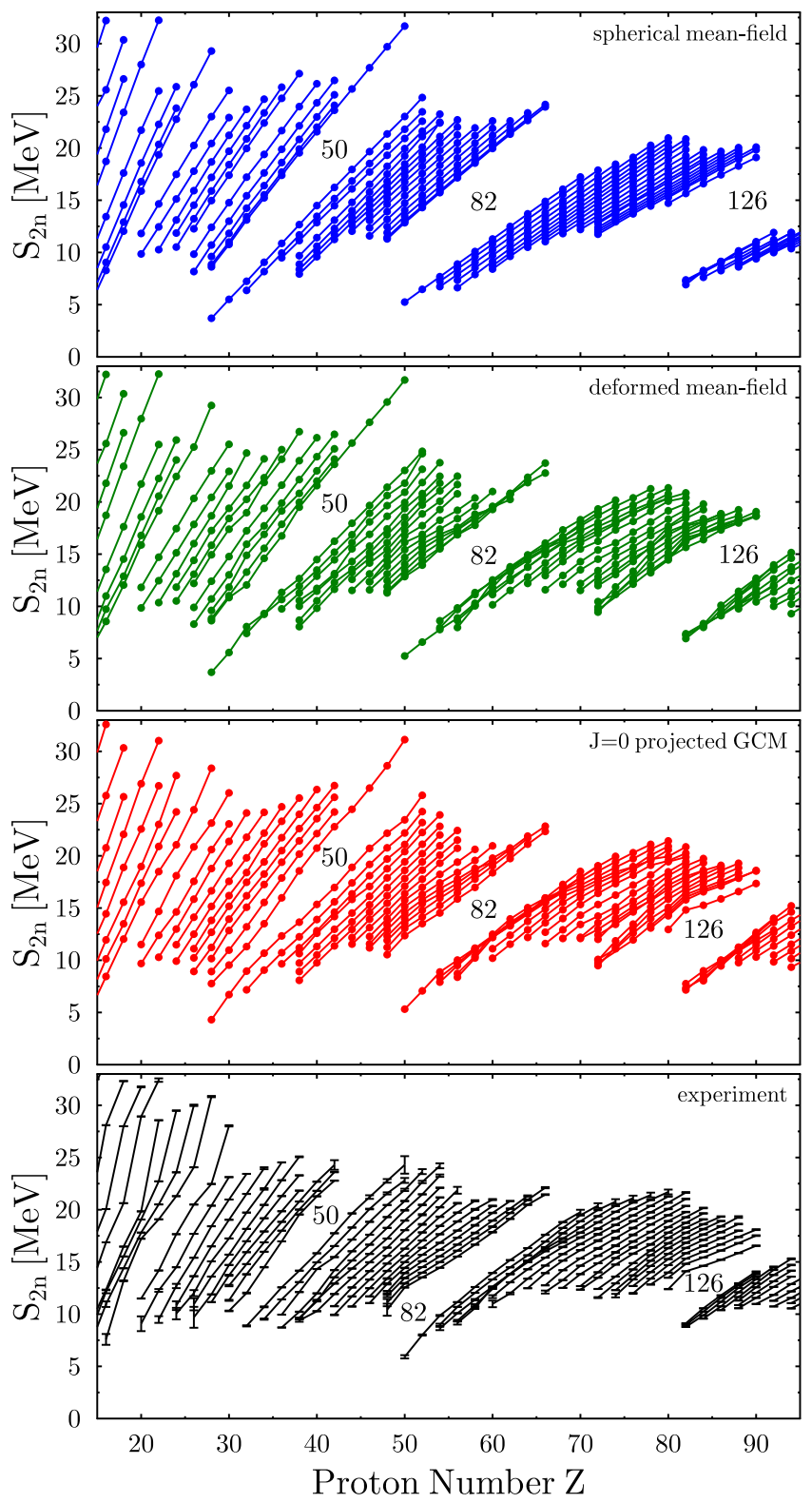

FIG. 4: (Color online) Two-neutron separation energy $S_{2 n}$ for even-even nuclei. Lines connect nuclei in isotopic chains

in many instances. As in the examples of the $Z=50$ and $N=50$ gaps, the magic nuclei remain spherical, but not all of their neighbors with \pm 2 protons are. The slope of the $S_{2 p}$ curves is also modified between the spherical shells: the energy gain due to deformation increases $S_{2 p}$ above magic numbers and decreases it below. Moreover, several small local gaps open between the spherical shell closures, and indicate deformed shell closures.

Correlations beyond mean-field amplify the effects brought by static deformation: the gaps at the magic numbers are reduced further. Confirming the discussion for the $Z=50$ chain, deformation and dynamical correlations reduce the gap in the $S_{2 p}$ energies at all shell closures to about half their value in spherical mean-field 
calculations. Also, the effect on energy of dynamical correlations is the largest at shell closures, where it rapidly changes from nucleus to nucleus, while it is nearly constant for open shell nuclei [21]. It is quite gratifying to see that adding the correlations brings the calculated values close to experiment. Note that a particularly strong quenching is visible for $Z=82$. The three calculations presented here are based on the same effective interaction which predicts a spherical single-particle spectrum with gaps much larger than those in the experimental $S_{2 p}$ energies that vary only by a few $100 \mathrm{keV}$ with the neutron number. Hence, the quenching observed in the experimental $S_{2 p}$ values results from the effects of deformation and of beyond mean-field correlations on the total binding energy and could be called "collectivity enhanced" quenching of the two-nucleon separation energies at shell closures, rather than quenching of the gaps in the spherical single-particle spectra.

Figure 4 shows the two-neutron separation energies $S_{2 n}$ for isotopic chains. Qualitatively, one finds a similar behavior as for the $S_{2 p}$ curves, and the quenching of the $S_{2 n}$ values from quadrupole correlations is even more pronounced. However, the overall agreement with experiment of the $S_{2 p}$ curves from the $J=0$ projected GCM is better than the one for $S_{2 n}$, as was already pointed out and discussed in detail in [20, 21]. The Skyrme interaction SLy4 used here (as all others we tested) seems to systematically overestimate neutron shell gaps, while proton shell gaps are described better.

The inclusion of beyond-mean field correlations has a smoothing effect on the variation of the two-particle separation energies as a function of $N$ or $Z$. The often unrealistic local fluctuations obtained without these correlations in our calculations or in those of deformed meanfield calculations by other groups [37], or from mean-fieldbased mass models such as the microscopic-macroscopic method [38] or the Brussels HFB mass fits [39] are to a large extent suppressed. Thus, the correlation energy added by fluctuations of the wave functions around the mean-field minima compensates the too abrupt shape changes (spherical to deformed, or prolate to oblate) obtained within a pure mean-field approach.

\section{SUMMARY}

The structural changes that occur in nuclei when going along isotopic or isotonic chains have been discussed on the basis of two-nucleon separation energies. We have shown that the effect that has been referred to as shell quenching and has sometimes been attributed to a reduction of the spherical gaps far from stability is adequately described by the introduction of dynamic collective quadrupole correlations. Much smoother trends with fewer kinks and discontinuities are obtained than in deformed mean-field calculations. In many instances, our calculated values are close to the data. Looking at the shell closures where the $S_{2 q}$ values exhibit dis- continuities, static mean-field deformations and dynamical correlations decrease systematically the amplitude of these gaps, and reduce them far from stability. Both effects are not related to a reduction of the spherical shell structure, rather both underline the importance of fluctuations around single mean-field configurations for a high-precision description of nuclear masses. Studies performed in restricted mass regions [20, 21, 36, 40, 41] have arrived at similar conclusions.

This study addresses also two present-day questions concerning mean-field models and effective interactions (or density functionals). For a long time, single particleenergies have been viewed as being not directly connected with experimental data because they are strongly renormalized by the coupling to different kinds of vibrations [18, 42, 43, 44]. However, it has been proposed recently [45] that this statement is too strong and that single-particle energies should be used in the fitting procedure of energy density functionals. This point has been convincingly demonstrated using specific data. Our analysis shows that one must carefully select the data relevant for adjusting single-particle levels and that the quenching of 2-particle separation energies should not be related to single particle properties. Our study also raises the question of whether all correlations should be included in an energy-density functional, or if some of them should be treated explicitly, beyond a mean-field approach. At the minimum, our study shows the interest of treating explicitly the correlations associated with fluctuations in collective degrees of freedom.

Finally, let us note that the relation between singleparticle energies and the evolution of 2-particle separation energies along $N$ or $Z$ is not a simpler problem in the shell model context. The definition which corresponds the most closely to a spherical mean-field single particle energy is the effective single-particle energy of Caurier et al. 11, 12], which includes the monopole shift of singleparticle energies, i.e. the terms of the interaction that correspond to the spherical mean field. A definition more directly related to nucleon transfer reactions is based on centroids of the strength distribution [14, 15, 16, 17]. Both are obtained from the averaging over correlated states which compromises their interpretation in terms of a pure configuration, and are neither a directly observable quantity. The main message of this contribution is probably that one should not try to carry out too indirect comparisons with experimental data and that the notion of single-particle states should be limited to the very few cases where correlation effects are weak.

\section{Acknowledgments}

MB thanks the organizers and participants of the workshop "Mass Olympics", held at ECT* Trento 26-30 May 2008 for many inspiring presentations and discussions. This research was supported in parts by the PAI-P5-07 of the Belgian Office for Scientific Policy, by the U.S. De- 
partment of Energy under Grant DE-FG02-00ER41132

(Institute for Nuclear Theory) and DE-AC02-06CH11357
(ANL).
[1] A. Poves, J. Sanchez-Solano, E. Caurier and F. Nowacki Nucl. Phys. A694, 157 (2001).

[2] M. Honma, T. Otsuka, B. A. Brown, and T. Mizusaki, Phys. Rev. C 69, 034335 (2004).

[3] M. Honma, T. Otsuka, B. A. Brown and T. Mizusaki, Eur. Phys. J. A25, s01, 499 (2005).

[4] D.-C. Dinca, et al., Phys. Rev. C 71, 041302(R) (2005)

[5] J. I. Prisciandaro et al., Phys. Lett. B510, 17 (2001)

[6] R. V. F. Janssens et al., Phys. Lett. B546, 55 (2002)

[7] J. Dobaczewski, I. Hamamoto, W. Nazarewicz, and J. A. Sheikh, Phys. Rev. Lett. 72, 981 (1994).

[8] D. Von-Eiff, H. Freyer, W. Stocker, and M. K. Weigel, Phys. Lett. B344, 11 (1995).

[9] G. A. Lalazissis, D. Vretenar, W. Pöschl, and P. Ring, Phys. Lett. B418, 7 (1998); Nucl. Phys. A632, 363 (1998); G. A. Lalazissis, D. Vretenar, and P. Ring, Phys. Rev. C 57, 2294 (1998).

[10] M. Bender, P.-H. Heenen, and P.-G. Reinhard, Rev. Mod. Phys. 75, 121 (2003).

[11] E. Caurier, F. Nowacki, and A. Poves, Eur. Phys. J. A15, 145 (2002).

[12] E. Caurier, G. Martinez-Pinedo, F. Nowacki, A. Poves, and A. P. Zuker, Rev. Mod. Phys. 77, 427 (2005).

[13] N. A. Smirnova, A. de Maesschalck, K. Heyde, AIP Proc. 819, 475 (2006).

[14] M. Baranger, Nucl. Phys. A149, 225 (1970).

[15] A. Signoracci and B. A. Brown, Phys. Rev. Lett. 99, 099201 (2007).

[16] B. P. Kay, S. J. Freeman, J. P. Schiffer, J. A. Clark, C. Deibel, A. Heinz, A. Parikh and C. Wrede, Phys. Lett. B 658, 216 (2008).

[17] A. Umeya, S. Nagai, G. Kaneko, and K. Muto Phys. Rev. C 77, 034318 (2008).

[18] H. S. Köhler, Phys. Scr. 10A, 81 (1974).

[19] J. Suhonen, From Nucleons to Nucleus, Springer Verlag, New York, Heidelberg, Berlin, (2006).

[20] M. Bender, G. F. Bertsch, and P.-H. Heenen, Phys. Rev. Lett. 94, 102503 (2005).

[21] M. Bender, G. F. Bertsch, and P.-H. Heenen, Phys. Rev. C 73, 034322 (2006).

[22] See EPAPS Document No. E-PRVCAN-73-865512 for tables with the intrinsic deformations and energies for 605 even-even nuclei. For more information on EPAPS, see http://www.aip.org/pubservs/epaps.html.

[23] E. Chabanat, P. Bonche, P. Haensel, J. Meyer, and R. Schaeffer, Nucl. Phys. A635, 231 (1998); A643, 441(E) (1998).

[24] P. Ring and P. Schuck, The Nuclear Many-Body Problem, Springer Verlag, New York, Heidelberg, Berlin, (1980).

[25] R. R. Rodríguez-Guzmán, J. L. Egido, and L. M. Robledo, Phys. Rev. C 62, 054319 (2000).

[26] L. M. Robledo, Int. J. Mod. Phys. E16, 337 (2007).

[27] M. Anguiano, J. L. Egido, and L. M. Robledo, Nucl.
Phys. A696, 467 (2001).

[28] J. Dobaczewski, M. V. Stoitsov, W. Nazarewicz, and P.G. Reinhard, Phys. Rev. C 76, 054315 (2007).

[29] D. Lacroix, T. Duguet, and M. Bender in preparation; M. Bender, T. Duguet, and D. Lacroix in preparation; T. Duguet, M. Bender, K. Bennaceur, D. Lacroix, and T. Lesinski, in preparation.

[30] P. Delahaye et al., Phys. Rev. C 74, 034331 (2006); M. Dworschak et al., Phys. Rev. Lett. 100, 072501 (2008); T. Eronen et al., Phys. Rev. Lett. 97, 232501 (2006); T. Eronen et al., Phys. Rev. Lett. 100, 132502 (2008); C. Gaulard et al., Nucl. Phys. A766, 52 (2006). C. Guénaut et al., Phys. Rev. C 75, 044303 (2007); U. Hager et al., Phys. Rev. Lett. 96, 042504 (2006); U. Hager et al., Nucl. Phys. A793, 20 (2007); U. Hager et al., Phys. Rev. C 75, 064302 (2007); A. Kankainen et al., Eur. Phys. J. A 29, 271 (2006); A. Kellerbauer et al., Phys. Rev. C 76, 045504 (2007); A. Martín et al., Eur. Phys. J. A 34, 341 (2007); S. Rinta-Antila et al., Eur. Phys. J. A 31, 1 (2007); S. Rahaman et al., Eur. Phys. J. A 32, 87 (2007); S. Rahaman et al., Eur. Phys. J. A 34, 5 (2007); P. Schury et al., Phys. Rev. C 75, 055801 (2007); G. Sikler et al., Nucl. Phys. A763, 45 (2005).

[31] J. Hakala et al., Phys. Rev. Lett. 101, 052502 (2008).

[32] G. Audi, A. H. Wapstra, and C. Thibault, Nucl. Phys. A729, 337 (2003).

[33] K.-H. Schmidt et al., Nucl. Phys. A318, 253 (1979).

[34] N. Zeldes, T. S. Dumitrescu, and H. S. Köhler, Nucl. Phys. A399, 11 (1983).

[35] D. Lunney, J. M. Pearson, and C. Thibault, Rev. Mod. Phys. 75, 1021 (2003).

[36] M. Bender, T. Cornelius, G.A. Lalazissis, J .A. Maruhn, W. Nazarewicz, and P.-G. Reinhard, Eur. Phys. J. A 14, $23(2002)$.

[37] J. Dobaczewski, M. Stoitsov and W. Nazarewicz, AIP Proc. 726, 51 (2004).

[38] P. Möller, J. R. Nix, W. D. Myers, and W. J. Swiatecki, Atom. Data Nucl. Data Tables 59, 185 (1995).

[39] S. Goriely, F. Tondeur, and J. M. Pearson, At. Data Nucl. Data Tables 77, 311 (2001).

[40] P. Fleischer, P. Klüpfel, T. Cornelius, T. J. Bürvenich, S. Schramm, J. A. Maruhn, and P.-G. Reinhard, Eur. Phys. J. A22, 363 (2004).

[41] A. Jungclaus and L. Egido, Phys. Scr. T 125, 53 (2006).

[42] I. Hamamoto, Phys. Rep. 10, 63 (1974).

[43] C. Mahaux, P. F. Bortignon, and R. A. Broglia, Phys. Rep. 120, 1 (1985).

[44] E. Litvinova and P. Ring, Phys. Rev. C 73, 044328 (2006).

[45] M. Zalewski, J. Dobaczewski, W. Satula, and T. R. Werner, Phys. Rev. C 77, 024316 (2008). 